\title{
Endocan: A new marker for cancer and a target for cancer therapy (Review)
}

\author{
JINGHUI YANG $^{1}$, QIWEI YANG ${ }^{1,2}$, SHAN YU $^{1}$ and XUEWEN ZHANG ${ }^{1}$ \\ ${ }^{1}$ China-Japan Union Hospital, Jilin University, Changchun, Jilin 130033; ${ }^{2}$ Central Laboratory, \\ Second Hospital, Jilin University, Changchun, Jilin 130041, P.R. China
}

Received December 15, 2014; Accepted February 5, 2015

DOI: $10.3892 /$ br. 2015.438

\begin{abstract}
Endocan, previously known as endothelial cell-specific molecule-1 (ESM-1), was cloned from the human umbilical vein endothelial cell cDNA library. Endocan is a novel ESM, and a $50 \mathrm{kDa}$ soluble proteoglycan. Endocan is secreted into the blood as the soluble proteoglycan, which is the form in the presence of chondroitin sulfate. In normal tissues, chondroitin sulfate/dermatan sulfate proteoglycan is expressed by endothelial cells (such as lung and kidney) and is overexpressed in several carcinoma endothelial cells. There are studies that identified high endocan expression in lung cancer, uterine cancer, kidney cancer, liver cancer, brain glioblastoma, breast cancer and other tumors. Tumor prognosis, metastasis and angiogenesis were shown to be associated with endocan expression. The majority of investigators believe that endocan regulates the tumor by tumor-associated inflammation, angiogenesis, lymphangiogenesis, the tumor cells themselves and other aspects. Endocan may be a new target for cancer therapy.
\end{abstract}

\section{Contents}

1. Introduction

2. Classic function of endocan

3. Endocan as a new marker of cancer and target for cancer therapy

4. Conclusion

Correspondence to: Dr Xuewen Zhang or Ms. Shan Yu, China-Japan Union Hospital, Jilin University, 126 XianTai Street, Changchun, Jilin 130033, P.R. China

E-mail: xuewenzhang01@126.com

E-mail: adashan521@sina.com

Key words: endocan, endothelial cell-specific molecule-1, tumor, cancer

\section{Introduction}

Endocan is a soluble dermatan sulfate proteoglycan (DSPG) secreted by endothelial cells with a molecular weight of $50 \mathrm{kDa}$. Endocan contains a core protein that consists of 165 amino acids and a single mucopolysaccharides chain that is covalently connected to the 137th (DS chains) serine residue. DS is a linear polysaccharide of $\mathrm{N}$-acetyl-galactosamine and iduronic acid (IdoA) or glucuronic acid disaccharide repeating unit. IdoA is an important component of the functional structure of endocan. In cancer patients, patients with sepsis and tissue samples of healthy volunteers, serum protein endocan is present in the form of a polysaccharide (1-4). Studies of the HUVEC vascular endothelial cell model show that the endocan expression level is extremely low, which cannot meet the requirements of scientific research (4). Therefore, Sarrazin et al (4) established a human embryo kidney 293 (HEK 293) cell model by gene recombination technology and demonstrated that the endocan proteoglycan molecules are capable of a high expression level in a model with a long cycle DS chain activity. Endocan expression was $>60$ times compared to the previous cell model $(174.3 \pm 49.7$ vs. $2.9 \pm 1.1 \mu \mathrm{g} / \mathrm{ml})$. The results for the study of endocan and other recently discovered glycosylated cytokines were provided favorable conditions.

The endocan-encoding gene is located on human chromosome 5 (5q11.2), which has three exons and two introns. Exons 1,2 and 3 are 362, 150 and 1,560 base pairs (bp) in length, respectively. The 1st and a section of the 2 nd outer exon can encode a particular amino acid terminus, which contains 110 amino acids and is cysteine-rich. The 2 nd outer exon encodes a particular region, which is rich in phenylalanine (F) (113FPFFQY118) and is associated with the function of endocan (5). The endocan protein carboxyl end is coded by the 3rd outer exon, which has 33 amino acids and has the early termination codon of this section and the $O$-glycosylation site for serine 137 (5). The endocan gene promoter is located in the flanking regions, and is from the $3,888 \mathrm{bp}$. The endocan gene has been cloned by investigators. The gene has a more typical TATA box, including a three Ets motifs ( -77 to -74 , -63 to -60 and +16 to +19$)$, a cyclic adenosine 3 ',5'-monophosphate (cAMP) responsive element-like element (-68 to -62) and a palindromic sequence (GCATGC) (-58 to -53) (6). The Ets motif in the literature is considered a cis-regulatory element (6). By way of mutation analysis, the first Ets motif is 
located between -77 to -74 and the cAMP responsive element is located between -68 to -62 , and they may be involved in regulation of high endothelial levels of endocan expression. DNA footprinting of the analysis for a complete promoter showed that the second Ets motif (-63 to -60$)$ and a palindromic sequence (-58 to -53$)$ can inhibit the expression of endocan, and therefore is a transcription inhibitor (6). Further Ets motifs are required for maximum activity by angiogenic factors, such as fms-like tyrosine kinase 1, tyrosine kinase with immunoglobulin-like and EGF-like domains (Tie), Tie-2 and vascular endothelial cadherin promoter (2). Another study identified that the endocan promoter has three GATA motifs, three activating protein-4 (AP4) motifs and two AP1 motifs (6). The gene can be cut to encode two endocan products with distinct expression: One is a mature endocan molecule, including a complete core protein with a F113 and F116 phenylalanine-rich region and a DSPG single-strand (proteoglycan-rich, single-stranded and phenylalanine pool are associated with endocan tumor promotion); the second product is an endocan molecule lacking the exon 2 promoter region, known as endocan $\Delta 2$. Depontieu et al (7) showed that endocan $\Delta 2$ does not promote tumor growth. Endocan $\Delta 2$ has the same endocan $\mathrm{N}$-and $\mathrm{C}$-terminus, but lacks the exon 2 encoding 50 amino acids. This exon 2 sequence affects protein oligomerization, thus losing the pro-tumor effect. In non-small cell lung cancer, there was no elevated content detected. This study shows that the main function of endocan is associated with the outer region of the 2 nd exon encoding the content-rich phenylalanine region and DS chains containing IdoA.

\section{Classic function of endocan}

Alternative splicing plays an important functional role in the regulation mechanism of endocan. Endocan expression is regulated by a series of cytokines or cell factors and the impact of inflammatory cytokines. A variety of inflammatory mediators, such as interleukin- 1 and tumor necrosis factor- $\alpha$, can promote endocan overexpression. Serum endocan expression levels in patients with more severe sepsis are relatively high; the release of inflammatory factors may prompt elevated serum endocan expression levels (8). Endocan gene expression levels may be due to the lipopolysaccharide (LPS) of the gram-negative bacterial cell wall, and thus increases. In vitro tests, including the addition of interleukin-1, tumor necrosis factor- $\alpha$ and/or following bacterial LPS, result in an increase of endocan secretion levels of endothelial cells. This increase can last $>72 \mathrm{~h}(9)$. The study also found that high levels of endocan expression correlate with certain angiogenic factors, such as vascular endothelial growth factor A (VEGF-A) and fibroblast growth factor-2 (FGF-2). Using cDNA probes and northern blot methods, Aitkenhead et al (10) found that the endocan gene expression levels increased by four times in cultured endothelial cells under stimulation of VEGF and FGF-2. This mechanism may be the endocan DS-chain binding the FGF-2 and FGF-2-dependent promotion of vascular endothelial cell proliferation, thereby promoting angiogenesis. VEGF-A regulated endocan expression through the phosphoinositide 3-kinase-protein kinase B (Akt) signaling pathway (11). This study identified that there are certain factors for the endocan upregulation, including the exact protein kinase $\mathrm{C}$ activator, VEGF-C, nuclear factor- $\kappa \mathrm{B}$ (NF- $\mathrm{\kappa B}$ ) and retinoic acid (2).

A study has shown that endocan can increase the hepatocyte growth factor/scatter factor (HGF/SF) chemotactic-mediated proliferation of human embryonic kidney cells and causes dose-dependent proliferation, but more importantly, is that the base of the non-sugar endocan form does not show this effect. Similar to endocan, HGF/SF cause purified chondroitin sulfate chains, which also has the effect of promoting cell proliferation. Endocan regulates the HGF/SF-mediated mitogenic behavior; this effect is not present in embryonic development and tissue damage following the repair process, but is also present in the process of tumor development. In inflammation and injury of human body fluids, the reaction of DSPGs plays an important role. In early embryonic development, HGF/SF aids the occurrence of organ morphogenesis for liver, kidney, lung, intestine and other tissues and organs (12).

Endocan has homology with the amino acid sequence of the adhesion molecules that participate in between cells, and between cells and the cell matrix. As the state of endothelial cells express low levels of adhesion molecules, such as intercellular adhesion molecule-1 (ICAM-1) and ICAM-2, this may help prevent spontaneous leukocyte adhesion to endothelial cells or leukocyte migration through the vessel wall. Certain investigators identified that in monocytes, lymphocytes and Jurket cells, endocan is involved in direct binding to integrins cluster of differentation 11a (CD11a)/CD18 [lymphocyte function-associated antigen-1 (LFA-1)] to prevent ICAM-I and LFA-1 binding by co-immunoprecipitation methods (3). The cell adhesion molecule of the LFA-1 integrin family is a heterodimeric transmembrane protein that consists of the $\alpha \mathrm{L} \beta 2$ chains, and its receptors include ICAM-1, ICAM- 2 and ICAM-3. In cell activity, the interaction of LFA-1 and ICAM-1 plays a crucial role, including participation in leukocyte adhesion, migration and activation. Experimental animal models confirmed that ICAM-1 and LFA-1 participate in the acute inflammatory response, ischemia/reperfusion injury, allograft and antitumor immunity. The results showed that endocan actively participates in the interaction of endothelial cells and leukocytes. The study by Béchard et al (3) confirmed that endocan acts on physical function or has a specific interaction with the LFA-1 integrin extracellular domain of Jurkat cells that rely on a combination of divalent cations, which are specificity, saturation and temperature sensitive. The binding sites of endocan and LFA-1 to ICAM-1 are different. LFA-1 and ICAM-1 bind, and ICAM-1 binds to endocan bound to LFA-I near I domain of CD11a. Endocan and ICAM-1 can be combined in Jurkat cells and have an influence on each other. Endocan may be involved in regulating the LFA-1/ICAM-1 pathway, resulting in decreased leukocyte activation by LFA-1, which may affect the lymphocyte homing to sites of inflammation and aggregation, and leukocyte adhesion and activation may be affected. Endocan expression in human lung and renal tissue may play a role in angiogenesis of organ-specific inflammation. Endocan is a novel endothelial cell activation marker. However, its involvement in acute renal transplant rejection is unclear. Studies have shown that endocan may reflect the degree of endothelial cell injury of renal allograft and it can be used as a highly sensitive and specific marker of acute rejection following renal transplantation (13). Endothelial cell-specific 


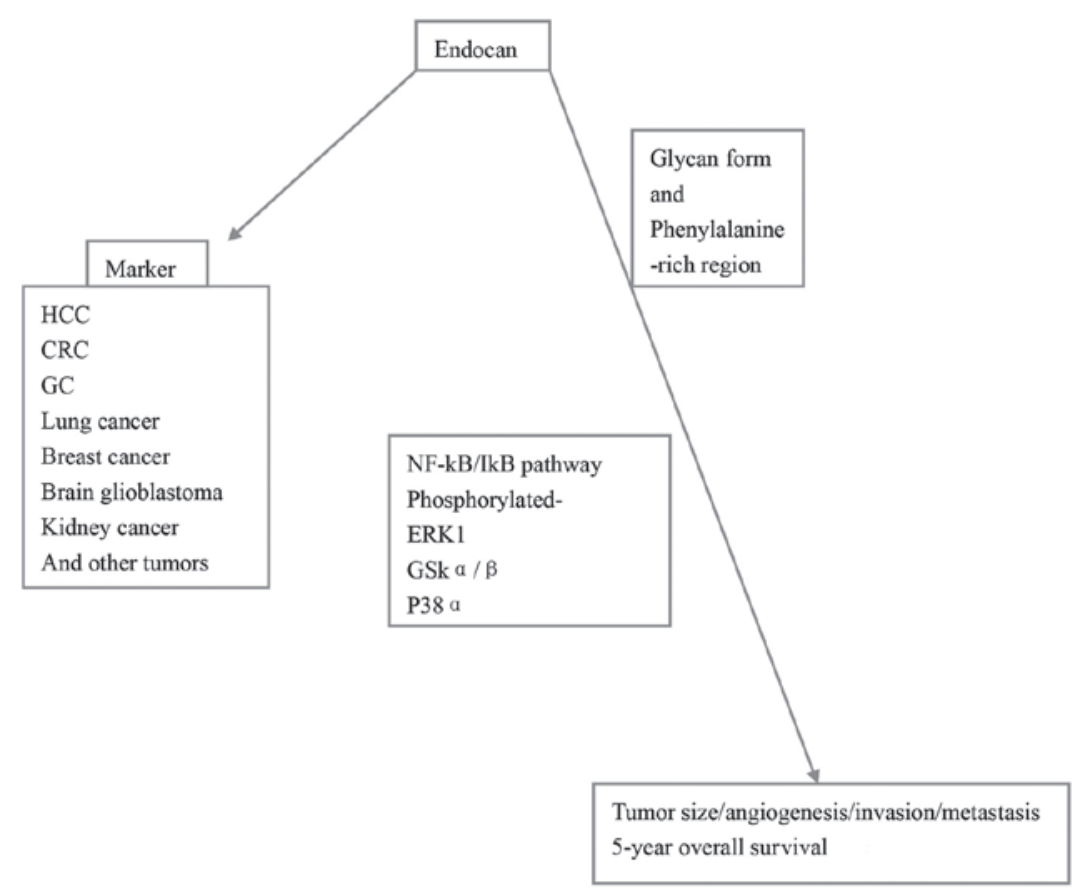

Figure 1. Endocan expression is high in hepatocellular carcinoma (HCC), colorectal cancer (CRC), gastric carcinoma (GC), lung cancer, breast cancer, brain glioblastoma, kidney cancer and other tumors. Endocan can be a tumor marker. Tumor prognosis, metastasis and angiogenesis have an association with endocan expression. Endocan has an association with the 5-year overall survival rate of patients. The glycan form and phenylalanine-rich region of endocan are the key effective sections through the nuclear factor- $\mathrm{\kappa B}(\mathrm{NF}-\mathrm{\kappa B}) / \mathrm{I} \mathrm{B}$, phosphorylated extracellular signal-regulated kinase 1 (ERK1), glycogen synthase kinase $\alpha / \beta(\mathrm{GSK} \alpha / \beta)$ and $\mathrm{P} 38 \alpha$ pathways.

molecule-1 (ESM-1) expression was observed in peripheral blood circulating endothelial cells and renal allografts following renal transplantation and was compared to the flow cytometry results of urine human leukocyte antigen-DR(+) lymphocytes. ESM-1 mRNA and protein expression increased significantly in patients with acute rejection $(\mathrm{P}<0.01)$. ESM-1 may reflect the degree of endothelial cell injury in renal allografts and may act as a highly sensitive and specific marker for acute rejection following renal transplantation (13). The study by Li et al (14) showed that peripheral blood endocan mRNA and protein expression in renal transplantation may reflect the endothelial cell injury severity.

In embryonic development, placenta formation, wound healing, inflammation and tumor formation in growth, angiogenesis and vascular growth are extremely important physiological or pathological processes. Numerous molecules have been known to participate in angiogenesis by inhibition or promotion, including the VEGF family, ephrin family, angiopoietin family, transforming growth factor- $\beta$ and platelet-derived growth factor. All of these factors coordinate and collaborate with a complementary functionality to form blood vessels when in the correct order. Angiogenesis is a complex process in particular, with numerous factors involved, but only VEGF has been shown to have a strict specificity. Initiation, development and maturation in the angiogenesis process are required, and VEGF is a marker of angiogenesis. In normal adult tissues, certain factors inhibit angiogenesis in a dominant state and the vascular endothelial cells in the stationary state. Under the stimulation of hypoxia and other factors, promoting angiogenesis factors, such as VEGF, cause the large release of endothelial cell activation and formation of new blood vessels.
Endocan is a common molecular property in vascular tissues, such as capillaries, arterioles and venules, and is expressed in vascular endothelial cells in normal tissues $(6,10)$. Endocan is highly restricted to endothelial cells, which may contribute to vascular endothelial cells, and endothelial cells play a role in dependent pathological processes (15). Thus, endocan can be used as a new endothelial cell activation marker (15). Although identified in numerous experiments, endocan and vascular proliferation showed a positive correlation, but the mechanism of endocan directly promoting angiogenesis remains to be revealed. Currently, the majority of investigators considered it to possibly be a pro-angiogenic mechanism through positive-feedback regulation. Vascular endothelial cells have been cultured so that HGF/SF, FGF-2 and VEGF promote the expression of endocan and each other (16), whilst accelerating endothelial cell proliferation and causing tubular structures to form more easily into the blood vessels (10). These results suggest that there may be a positive-feedback mechanism in this process, and that tumor epithelial cells secrete growth factors that stimulate endothelial cell proliferation and secretion of endocan. Endocan stimulates tumor cell proliferation and secretion of growth factors more, and further promotes the proliferation of blood vessels (12).

\section{Endocan as a new marker of cancer and target for can- cer therapy}

There are studies that have identified that endocan expression is high in lung cancer, uterine cancer, kidney cancer, liver cancer, brain glioblastoma, breast cancer and other tumors (Fig. 1) (17,18). Endocan may be a new target for cancer 
therapy $(17,19)$. Endocan is overexpressed in tumor cells and promotes tumor growth (5). One study showed that HEK 293 cells overexpressing endocan were shown to form tumors in severe combined immunodeficiency (SCID) mice. Two additional HEK 293-endocan cell clones were injected in eight mice and this also formed tumors, evidently avoiding clonal selection artifacts. Circulating levels of endocan increased over time and correlated positively with tumor size (5). The glycan from endocan is required for action (5). A nonglycanated form was generated by mutagenesis of serine 137 to alanine (endocan/S137A). The HEK 293 cell clones expressing this cDNA mutant were selected and screened for mutant endocan secretion. Each of the two endocan/S137A clones that secreted $2,023 \mathrm{ng} / \mathrm{ml} \pm 360 \mathrm{ng}$ endocan $/$ day $/ 10^{6}$ cells were subcutaneously injected into groups of four SCID mice in three separate experiments and were systematically compared to the positive control group receiving endocan-expressing clones. HEK 293 cells consistently develop a tumor (cumulative frequency of 32 tumors among 34 mice in eight distinct experiments). However, endocan/S137A-expressing clones did not induce tumor formation (0 tumor/12 mice) (5). The other key requirement for correct endocan action is the phenylalanine-rich region (5). The study mutated phenylalanine and subsequently, the HEK 293 cells did not form any tumors despite the presence of the glycan (5). Immunofluorescent staining showed strong ESM-1 expression in the cytoplasm of tumor cells in hepatocellular carcinoma (HCC) tissues when compared to normal tissues (20). A previous study analyzed the tumor tissues and surrounding non-cancerous hepatic parenchyma from 42 primary HCC patients. Endothelial cells were isolated using magnetic microbeads conjugated with anti-CD31 and endocan expression was evaluated by reverse transcription-quantitative polymerase chain reaction, western blot analysis and immunohistochemistry. Endocan was significantly overexpressed in endothelial cells isolated from HCC tumors compared to the corresponding non-cancerous liver tissues (21). ESM-1 is also expressed at the periphery of the tumor cells in HCC tissue. The study compared serum ESM-1 in normal subjects and in patients with HCC. The ESM-1 levels were significantly higher in HCC patients compared to the normal subjects. The specificity of ESM-1 was $80.8 \%$ and sensitivity was $83.8 \%$ at the cutoff value. For $\alpha$-fetoprotein, the specificity was $90.8 \%$ and sensitivity was $56.8 \%$ (cutoff value $20.0 \mathrm{ng} / \mathrm{ml}$ ) (20). The mRNA level of ESM-1 in tissues was correlated with the tumor-node-metastasis phase of HCC patients, tumor vascular invasion and metastasis (22). Ziol et al (23) showed that the interstitial vascular endothelial cells of the endocan-expressing HCC tumor biopsy samples have an independent predictive value for the radiofrequency ablation in patients with early recurrence. Experiments and human studies indicate that endocan expression in tumor angiogenesis reflects the angiogenesis and tumor invasion (21). ESM-1 mRNA levels were much higher in metastatic compared to non-metastatic liver tumor samples. Serum ESM-1 levels were significantly higher in HCC patients compared to normal subjects. Cell viability was evaluated with the WST-1 reagent. At $24 \mathrm{~h}$ after assessing ESM-1 small interfering (si)RNA expression, cell viability was reduced $\leq 11 \%$. The expression levels of I $\mathrm{B}$ and NF- $\kappa \mathrm{B}$ p 65 , members of the $\mathrm{NF}-\kappa \mathrm{B}$ subfamily, were evaluated by immunoblotting. Increased phospho-I $\kappa \mathrm{B}$ and downregulated NF- $\mathrm{B}$ p65 expression levels suggested that NF- $\mathrm{B}$ is suppressed in ESM-1 siRNA-expressed SK-Hep1 cells, indicating that ESM-1 was able to increase the SK-Hep1 cell survival rate via the I $\mathrm{B}$-dependent NF- $\kappa \mathrm{B}$ pathway. Expression of ESM-1 siRNA inhibited HCC cell migration and invasion (20). Studies have shown that endocan immune response correlates with colon tumor size, depth of invasion, lymph node metastasis, distant metastasis and Dukes' staging, and is an independent prognostic factor in disease recurrence (24). Phosphorylated extracellular signal-regulated kinase 1 (ERK1), glycogen synthase kinase (GSK) $\alpha / \beta, p 38 \alpha / \delta$, ribosomal S6 kinase 1 ( $R S K 1), A k t 1$, pan-Akt and heat-shock protein 27 (HSP27) were highly expressed in cells transfected with the control siRNA. By contrast, phosphorylated ERK1, $G S K \alpha / \beta$ and $p 38 \alpha$ expression levels were decreased and phospho-p38ס, -RSK1, -Akt1, -pan-Akt and -HSP27 were not detected in ESM-1 knockdown cells (25). In colorectal cancer, the interactions of endocan, $\mathrm{NF}-\kappa \mathrm{B}$ activation and $\mathrm{NF}-\kappa \mathrm{B}$ promoter are involved in cell survival, cell cycle progression, migration, invasion and tumor invasion epithelial-mesenchymal transition (EMT) (25). Akt-dependent $N F-\kappa B$ is suppressed in ESM-1 siRNA-expressing COLO205 cells, indicating that ESM-1 increased the COLO205 cell survival rate via the Akt-dependent $\mathrm{NF}-\kappa \mathrm{B} / \mathrm{I} \kappa \mathrm{B}$ pathway (25). The c-Jun N-terminal kinase, ERK and p38 pathways are not directly associated with the suppression of survival in ESM-1 siRNA-expressing COLO205 cells, indicating that ESM-1 may affect COLO205 cell survival via the Akt-dependent $\mathrm{NF}-\kappa \mathrm{B} / \mathrm{I} \kappa \mathrm{B}$ pathway, rather than other survival-related pathways (25). ESM-1 gene silencing regulates cell cycle arrest by phosphatase and tensin homolog (PTEN) induction, and PTEN-induced growth suppression in COLO205 cells (25). Endocan is known to influence cell invasion (25), and ESM-1 siRNA-expressing cells had a cell invasion rate of 1.35 -fold less than those expressing control siRNA. Therefore, the inhibition of $E S M-1$ by its siRNA expression in colorectal cancer cells inhibits cell invasion (25). ESM-1 siRNA expression inhibits the metastatic process through regulation of $M M P$ and $E M T$-related gene expression (25). For early stage colorectal cancer cases, 5-year overall survival rates were $100 \%(36 / 36)$ and $80 \%(12 / 15)$ for the patients with low and high serum ESM-1 levels, respectively, and there was a significant difference between the two groups (26). In gastric carcinoma (GC), ESM-1 mRNA was significantly higher in tumor compared to non-tumor tissues (26). The ESM-1 protein was expressed by tumor epithelium in 92 of $159(58 \%)$ cases, particularly in the tumor cell plasma and the periphery of the tumor cells, which were lower expressed relative to the matched histopathologically negative samples $(13 / 40,33 \%)$ that were obtained near the excision site from the same tumor patients (26). The serum levels were significantly higher in patients with late stage GC (stage III and IV) than those with early stage GC (stage I and II) $(87.23 \pm 15.1$ and $80.25 \pm 15.6 \mathrm{pg} / \mathrm{ml}$, respectively; $\mathrm{P}=0.018)(27)$. The 5-year overall survival rates were 85 (45/53) and $51 \%$ (31/61) for the patients with low and high serum ESM-1 levels, respectively, and there was a significant difference between the two groups $(\mathrm{P}<0.001)$. For early stage GC cases, 5-year overall survival rates were $86 \%(24 / 28)$ and $59 \%(16 / 27)$ for the patients with low and high serum ESM-1 levels, respectively (27). Anti-ESM-1 treatment inhibited tumor cells and vascular endothelial cells proliferation (26). 


\section{Conclusion}

Angiogenesis and tumor immunological characteristics have great significance in cancer development and metastasis. Current research indicates that endocan does not only affect the immunological characteristics of tumor vascular endothelial cells, but also has a close association with tumor angiogenesis. Therefore, endocan may be a tumor marker and possible new target for cancer therapy. Further research for endocan antitumor treatment methods is required. The research remains in the experimental stage and in theoretical studies; however, with understanding of its structure and function, endocan as a target antitumor therapy is bound to make a breakthrough.

\section{References}

1. Leroy X, Aubert S, Zini L, Franquet H, Kervoaze G, Villers A, Delehedde M, Copin MC and Lassalle P: Vascular endocan (ESM-1) is markedly overexpressed in clear cell renal cell carcinoma. Histopathology 56: 180-187, 2010.

2. Sarrazin S, Adam E, Lyon M, Depontieu F, Motte V, Landolfi C, Lortat-Jacob H, Bechard D, Lassalle P and Delehedde M: Endocan or endothelial cell specific molecule-1 (ESM-1): A potential novel endothelial cell marker and a new target for cancer therapy. Biochim Biophys Acta 1765: 25-37, 2006.

3. Béchard D, Scherpereel A, Hammad H, Gentina T, Tsicopoulos A, Aumercier M, Pestel J, Dessaint JP, Tonnel AB and Lassalle P: Human endothelial-cell specific molecule-1 binds directly to the integrin CD11a/CD18 (LFA-1) and blocks binding to intercellular adhesion molecule-1. J Immunol 167: 3099-3106, 2001.

4. Sarrazin S, Lyon M, Deakin JA, Guerrini M, Lassalle P, Delehedde $\mathrm{M}$ and Lortat-Jacob H: Characterization and binding activity of the chondroitin/dermatan sulfate chain from Endocan, a soluble endothelial proteoglycan. Glycobiology 20: 1380-1388, 2010.

5. Scherpereel A, Gentina T, Grigoriu B, Sénéchal S, Janin A, Tsicopoulos A, Plénat F, Béchard D, Tonnel AB and Lassalle P: Overexpression of endocan induces tumor formation. Cancer Res 63: 6084-6089, 2003

6. Tsai JC, Zhang J, Minami T, Voland C, Zhao S, Yi X, Lassalle P, Oettgen P and Aird WC: Cloning and characterization of the human lung endothelial-cell-specific molecule-1 promoter. J Vasc Res 39: 148-159, 2002.

7. Depontieu F, Grigoriu BD, Scherpereel A, Adam E, Delehedde M, Gosset $\mathrm{P}$ and Lassalle P: Loss of Endocan tumorigenic properties after alternative splicing of exon 2. BMC Cancer 8: 14, 2008.

8. Scherpereel A, Depontieu F, Grigoriu B, Cavestri B Tsicopoulos A, Gentina T, Jourdain M, Pugin J, Tonnel AB and Lassalle P: Endocan, a new endothelial marker in human sepsis. Crit Care Med 34: 532-537, 2006.

9. Grigoriu BD, Depontieu F, Scherpereel A, Gourcerol D, Devos P, Ouatas T, Lafitte JJ, Copin MC, Tonnel AB and Lassalle P: Endocan expression and relationship with survival in human non-small cell lung cancer. Clin Cancer Res 12: 4575-4582, 2006

10. Aitkenhead M, Wang SJ, Nakatsu MN, Mestas J, Heard C and Hughes CCW: Identification of endothelial cell genes expressed in an in vitro model of angiogenesis: Induction of ESM-1, (beta)ig-h3, and NrCAM. Microvasc Res 63: 159-171, 2002.
11. Rennel E, Mellberg S, Dimberg A, et al: Endocan is a VEGF-A and PI3K regulated gene with increased expression in human renal cancer. Exp Cell Res 313: 1285-1294, 2007.

12. Béchard D, Gentina T, Delehedde M, et al: Endocan is a novel chondroitin sulfate/dermatan sulfate proteoglycan that promotes hepatocyte growth factor/scatter factor mitogenic activity. J Biol Chem 276: 48341-48349, 2001.

13. Li S, Wang L, Wang C, et al: Detection on dynamic changes of endothelial cell specific molecule 1 in acute rejection after renal transplantation. Urology 80: 738. e1-8, 2012.

14. Li S, Liang P, Zhao Y, et al: Detection and mechanism of action of ESM-1 in rat kidney transplantation under various immune states. Cell Immunol 283: 31-37, 2013.

15. Bechard D, Meignin V, Scherpereel A, Oudin S, Kervoaze G, Bertheau P, Janin A, Tonnel A and Lassalle P: Characterization of the secreted form of endothelial-cell-specific molecule 1 by specific monoclonal antibodies. J Vasc Res 37: 417-425, 2000.

16. Gerritsen ME, Tomlinson JE, Zlot C, Ziman M and Hwang S: Using gene expression profiling to identify the molecular basis of the synergistic actions of hepatocyte growth factor and vascular endothelial growth factor in human endothelial cells. Br J Pharmacol 140: 595-610, 2003.

17. Abid MR, Yi X, Yano K, Shih SC and Aird WC: Vascular endocan is preferentially expressed in tumor endothelium. Microvasc Res 72: 136-145, 2006.

18. Maurage CA, Adam E, Minéo JF, Sarrazin S, Debunne M, Siminski RM, Baroncini M, Lassalle P, Blond S and Delehedde M: Endocan expression and localization in human glioblastomas. J Neuropathol Exp Neurol 68: 633-641, 2009.

19. Lassalle P, Molet S, Janin A, Heyden JV, Tavernier J, Fiers W, Devos R and Tonnel AB: ESM-1 is a novel human endothelial cell-specific molecule expressed in lung and regulated by cytokines. J Biol Chem 271: 20458-20464, 1996.

20. Kang YH, Ji NY, Lee CI, et al: ESM-1 silencing decreased cell survival, migration, and invasion and modulated cell cycle progression in hepatocellular carcinoma. Amino Acids 40: 1003-1013, 2011.

21. Chen LY, Liu X, Wang SL and Qin CY: Over-expression of the Endocan gene in endothelial cells from hepatocellular carcinoma is associated with angiogenesis and tumour invasion. $\mathrm{J}$ Int Med Res 38: 498-510, 2010.

22. Xiang X, Zhao WB and Wang X: Expression of ESM-1 in hepatocellular carcinoma is associated with angiogenesis and tumor invasion. Zhonghua Gan Zang Bing Za Zhi 17: 661-664, 2009 (In Chinese).

23. Ziol M, Sutton A, Calderaro J, et al: ESM-1 expression in stromal cells is predictive of recurrence after radiofrequency ablation in early hepatocellular carcinoma. J Hepatol 59: 1264-1270, 2013.

24. Kim JH, Park MY, Kim CN, Kim KH, Kang HB, Kim KD and Kim JW: Expression of endothelial cell-specific molecule-1 regulated by hypoxia inducible factor- $1 \alpha$ in human colon carcinoma: Impact of ESM-1 on prognosis and its correlation with clinicopathological features. Oncol Rep 28: 1701-1708, 2012.

25. Kang YH, Ji NY, Han SR, et al: ESM-1 regulates cell growth and metastatic process through activation of NF- $\kappa \mathrm{B}$ in colorectal cancer. Cell Signal 24: 1940-1949, 2012.

26. Liu N, Zhang LH, Du H, Hu Y, Zhang GG, Wang XH, Li JY and Ji JF: Overexpression of endothelial cell specific molecule-1 (ESM-1) in gastric cancer. Ann Surg Oncol 17: 2628-2639, 2010.

27. Lv Z, Fan Y, Chen H and Zhao D: Endothelial cell-specific molecule-1: A potential serum marker for gastric cancer. Tumour Biol 35: 10497-10502, 2014. 